\section{Ungewöhnliche Ursache einer Nahrungsmittelallergie}

\begin{abstract}
Ab und zu tauchen Berichte auf über die Entstehung von Nahrungsmittelallergien durch nicht intestinale Exposition mit einem bestimmten Allergen. Eine Studie aus Japan berichtet über eine Sensibilisierung gegen Weizen, die durch eine Gesichtsseife hervorgerufen wurde.
\end{abstract}

$B^{e}$ ereits früher gab es Berichte über eine Allergieentstehung gegen Erdnuss durch entsprechend allergenhaltige Hautpflegemittel. Japanische Allergologen konnten nun bei japanischen Frauen die Entstehung einer Weizenmehlallergie über diesen Sensibilisierungsweg sehr wahrscheinlich belegen. Schon 2010 wurde in Japan zunächst anekdotisch über Frauen publiziert, die nach Benutzung einer Seife mit hydrolysiertem Weizenmehleiweiß eine anstrengungsinduzierte Anaphylaxie nach Weizen gezeigt hatten.

Die landesweite Fall-Kontroll-Studie von Fukotomi et al. konnte diese Beobachtung nun erhärten: 157 erwachsene Frauen mit und 449 ohne eine Weizenmehlallergie wurden ausführlich interviewt. Dabei zeigte sich, dass eine Anwendung dieser speziellen Seife signifi- kant mit einem erhöhten Risiko für eine Weizenmehlallergie assoziiert war (Odds Ratio: 2,6, 95\%-Konfidenzintervall 1,25,7). Auch hatten die betroffenen Frauen diese Seife häufiger benutzt als die Kontrollgruppe (11\% vs. $6 \%$ ), was schon recht deutlich auf einen Zusammenhang zwischen der nicht intestinalen Sensibilisierung und der anschließenden Nahrungsmittelallergie hinweist.

Der Hersteller zog übrigens nach Bekanntwerden der Zusammenhänge diese Seife vom Markt zurück. Wieso speziell diese Seife so intensiv sensibilisierend wirkte, konnte bislang nicht eindeutig geklärt werden, ebenso wenig die zugrunde liegenden immunologischen Mechanismen. Eventuell könnte das komplexe produktionstechnische Verfahren hier eine Rolle spielen.

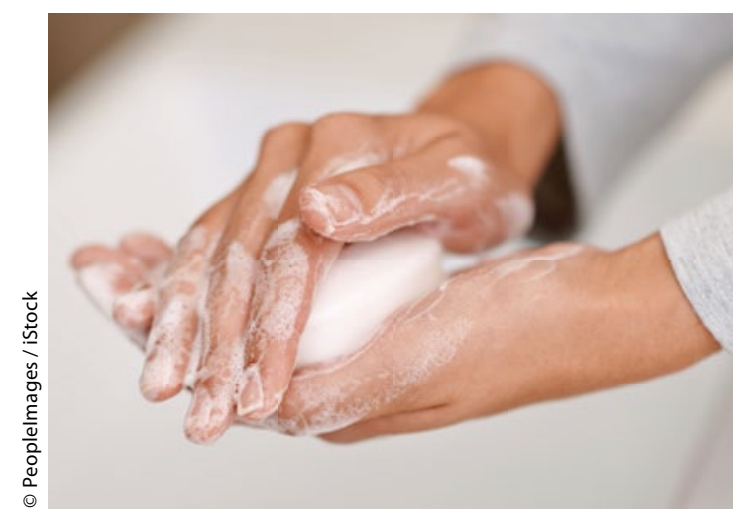

Die in einer Seife enthaltenen Stoffe führten zu einer Lebensmittelallergie.

Fukotomi Y et al. Epidemiological link between wheat allergy and exposure to hydrolyzed wheat protein in facial soap. Allergy $2014 ; 69$ : 1405-11

\section{Kommentar:}

Auch Produkte, in denen scheinbar rein natürliche Inhaltsstoffe wie Weizenmehleiweiß verarbeitet sind, können Auslöser oder Ursache einer Lebensmittelallergie bis hin zur Anaphylaxie sein - selbst dann, wenn sie primär nicht oral aufgenommen wurden.

Dr. Ulrich Mutschler

\title{
Der unkontrollierte Status epilepticus
}

\section{Was tun bei refraktärem Status epilepticus? Dieser Frage gingen zwei US- amerikanischen Studien nach, die beide in derselben Ausgabe der Zeitschrift Pediatric Critical Care Medicine erschienen sind.}

Ein in refraktärer Status epilepticus (RSE) konnte nicht durch First- und SecondLine-Antiepileptika beendet werden und persistiert länger als 30-60 Minuten. Ein Super-RSE liegt bei Anfallstätigkeit trotz über 24 Stunden induziertem Koma vor. Was sind nun die therapeutischen Empfehlungen? Eine Arbeitsgruppe aus Boston hat sich auf die Suche nach publizierten, harten Daten gemacht $[1,2]$.

Von 9.003 im Rahmen einer systematischen Literaturrecherche analysierten Studien zur besten Evidenz des RSE bei Kindern erfüllten schließlich nur 16 Studien (645 Patienten) die geforderten Einschlusskriterien. Wurde Midazolam als initiale Substanz eingesetzt kam es in
$76 \%$ zur klinischen Anfallskontrolle; dies durchschnittlich nach 41 Minuten. Als Folgesubstanzen wurden bei Midazolamversagen gewöhnlich Barbiturate verwendet. Nach 23 Stunden wurde im Durchschnitt ein „burst suppression“-Muster erreicht; die Effektivität lag bei $65 \%$. Danach kamen inhalative Anästhetika, Ketamin und Hypothermie zum Einsatz.

1. Bennet KS. Pediatric refractory status epilepticus: many more questions than answers. Pediatr Crit Care Med 2014;15:674-6

2. Wilkes $R$ et al. Intensive care treatment of uncontrolled status epilepticus in children: systematic literature search of midazolam and anesthetic therapies. Pediatr Crit Care Med 2014;15:632-9

\section{Kommentar:}

Die Mortalität des RSE bei Kindern liegt bei bis zu $30 \%$. Rund $50 \%$ der Überlebenden haben neurologische Defizite. Zudem sollte beim RSE das Ziel nicht nur das Sistieren der klinischen Anfälle, sondern auch der EEGPathologie sein. Das Management des RSE ist eine "vollkommen evidenzfreie Zone“. In diesem "Nichtwissen" ist es weniger wichtig womit, sondern wie schnell, mit welchen Dosen und Risiken der RSE behandelt wird. Wir brauchen dringend einheitliche Protokolle mit festgelegten Zeiten und Dosisvorgaben, idealerweise im Rahmen multizentrischer Therapiestudien. Auch wenn Propofol als kontinuierliche Applikation aufgrund des sehr seltenen aber schwerwiegenden "Propofol-Infusionssyndroms" in Kinderstudien nicht eingeht beziehungsweise problematisch ist, ist diese Substanz dennoch gut steuerbar und hoch effektiv. Die frühe Einzelgabe sollte meines Erachtens nicht unversucht bleiben - denn: time is brain!

Dr. Thomas Hoppen 